\title{
Survival benefits of hypofractionated radiotherapy combined with temozolomide or temozolomide plus bevacizumab in elderly patients with glioblastoma aged $\geq$ 75 years
}

Makoto Ohno ${ }^{1}$, Yasuji Miyakita ${ }^{1}$, Masamichi Takahashi ${ }^{1}$, Hiroshi Igaki ${ }^{2}$, Yuko Matsushita ${ }^{1}$, Koichi Ichimura ${ }^{3}$ and Yoshitaka Narita ${ }^{1 *}$

\begin{abstract}
Background and purpose: The purpose of this study was to evaluate the outcomes of elderly patients (aged $\geq 75$ years) with newly diagnosed glioblastoma (GBM), who were treated with hypofractionated radiotherapy comprising $45 \mathrm{~Gy}$ in 15 fractions combined with temozolomide (TMZ) or TMZ and bevacizumab (TMZ/Bev).

Materials and methods: Between October 2007 and August 2018, 30 patients with GBM aged $\geq 75$ years were treated with hypofractionated radiotherapy consisting of $45 \mathrm{~Gy}$ in 15 fractions. Twenty patients received TMZ and 10 received TMZ/Bev as upfront chemotherapy. O-6-methylguanine DNA methyltransferase (MGMT) promoter methylation status was analyzed by pyrosequencing. The cutoff value of the mean level of methylation at the $16 \mathrm{CpG}$ sites was $16 \%$.

Results: Median overall survival (OS) and progression-free survival (PFS) were 12.9 months and 9.9 months, respectively. The 1-year OS and PFS rates were 64.7 and 34.7\%, respectively. Median OS and PFS did not differ significantly between patients with MGMT promoter hypermethylation $(N=11)$ and those with hypomethylation $(N=16)(17.4 \mathrm{vs} .11 .8$ months, $p=0.32$; and 13.1 vs. 7.3 months, $p=0.11$, respectively). The median OS and PFS were not significantly different between TMZ $(N=20)$ and TMZ/Bev $(N=10)$ chemotherapy (median OS: TMZ 12.9 months vs. TMZ/Bev 14.6 months, $p=0.93$, median PFS: TMZ 8.5 months vs TMZ/Bev 10.0 months, $p=0.64$, respectively). The median time until Karnofsky performance status (KPS) score decreasing below 60 points was 7.9 months. The best radiological responses included 11 patients with a partial response (36.7\%). Grade 3/4 toxicities included leukopenia in 15 patients (50\%), anorexia in 4 (13.3\%), and hyponatremia during concomitant chemotherapy in 3 (10\%).
\end{abstract}

Conclusion: Our hypofractionated radiotherapy regimen combined with TMZ or TMZ/Bev showed benefits in terms of OS, PFS, and KPS maintenance with acceptable toxicities in elderly patients with GBM aged $\geq 75$ years.

Keywords: Elderly glioblastoma, Hypofractionated radiotherapy, Temozolomide, Bevacizumab, Vulnerability

\footnotetext{
* Correspondence: yonarita@ncc.go.jp

1 Department of Neurosurgery and Neuro-Oncology, National Cancer Center

Hospital, 5-1-1, Tsukiji, Chuo-ku, Tokyo 104-0045, Japan

Full list of author information is available at the end of the article
}

C The Author(s). 2019 Open Access This article is distributed under the terms of the Creative Commons Attribution 4.0 International License (http://creativecommons.org/licenses/by/4.0/), which permits unrestricted use, distribution, and reproduction in any medium, provided you give appropriate credit to the original author(s) and the source, provide a link to the Creative Commons license, and indicate if changes were made. The Creative Commons Public Domain Dedication waiver (http://creativecommons.org/publicdomain/zero/1.0/) applies to the data made available in this article, unless otherwise stated. 


\section{Introduction}

Glioblastoma (GBM) is the most aggressive type of primary brain tumor. It predominantly affects elderly patients; the median age at diagnosis is 67 years, and $16.3 \%$ of patients diagnosed with GBM in Japan are older than 75 years [1]. Age is one of the most consistently reported prognostic factors and survival is shown to decline with increasing age [2, 3]. The 1-year relative survival rates in the United States are 29.3 and $12.2 \%$ for patients with GBM aged 65-74, and those aged $\geq 75$ years, respectively [4]. Thus, the outcome of older patients is extremely poor.

In the management of elderly patients with GBM, hypofractionated radiotherapy has been investigated due to its advantage of having a shorter treatment time [5]. A trial comparing treatment with 40 Gy in 15 fractions to standard radiotherapy consisting of $60 \mathrm{~Gy}$ in 30 fractions did not show any differences in overall survival (OS), suggesting that the short-course radiation therapy (RT) was not an inferior treatment option [6]. The Nordic trial, which randomly assigned patients with newly diagnosed GBM who were aged 60 years and older to receive TMZ monotherapy, hypofractionated radiotherapy of $34 \mathrm{~Gy}$ in 10 fractions, or standard radiotherapy of 60 Gy in 30 fractions, demonstrated that hypofractionated radiotherapy produced survival rates that were similar to those in patients receiving standard radiotherapy [7].

In 2017, Perry et al. reported a phase III study showing that the addition of TMZ to short-course radiotherapy (40 Gy in 15 fractions) resulted in longer survival than radiotherapy alone in patients aged 65 years or older, thereby establishing TMZ combined with radiotherapy at $40 \mathrm{~Gy}$ in 15 fractions as the standard therapy in elderly patients with GBM [8]. However, in this study the median age was 73 years, and only $30 \%$ of patients were aged > 75 years; therefore, the survival impact of this chemoradiation therapy regimen on older patients $(>75$ years) remains unclear [9]. Moreover, there have been no comparative studies comparing the regimen of $40 \mathrm{~Gy}$ in 15 fractions or other hypofractionated regimens in combination with TMZ, therefore, optimal dose-fractionation schedules in combination with TMZ remains unclear [10]. Furthermore, the advantage of addition of bevacizumab (Bev) to TMZ combined with hypofractionated radiotherapy has not been fully evaluated [11-13]. Therefore, optimal treatment remains unclear especially in patients aged $\geq 75$ years.

We hypothesized that TMZ combined with doseintensified RT with the same fractionation as the regimen of $40 \mathrm{~Gy}$ in 15 fractions may improve patient outcomes. In addition, because elderly patients are known to have more angiogenic tumors than those who are younger [14], upfront addition of Bev to TMZ may have an advantage. We determined a dosage of $45 \mathrm{~Gy}$ in 15 fractions as our exploratory regimen based on a previous report that used a 15-fraction schedule [15] combined with TMZ, or TMZ plus Bev (TMZ/Bev).

In this study, we retrospectively analyzed the treatment outcomes of patients with newly diagnosed GBM aged 75 years of age or older, treated with hypofractionated radiotherapy consisting of $45 \mathrm{~Gy}$ in 15 fractions, combined with TMZ or TMZ/Bev to evaluate the feasibility of this treatment among a cohort of older patients (aged $\geq 75$ years).

\section{Patients and methods \\ Patient characteristics}

Forty-two patients aged $\geq 75$ years were diagnosed with GBM at our center between October 2007 and August 2018. Among these patients, 12 were excluded from this study for various reasons (RT alone in 5 patients, RT consisting of $60 \mathrm{~Gy}$ in 30 fractions in 4, TMZ alone in 1, $\mathrm{RT}$ and Bev, and refusal of therapy in 1). The remaining 30 patients who underwent initial surgery followed by hypofractionated radiotherapy consisting of $45 \mathrm{~Gy}$ in 15 fractions combined with TMZ or TMZ/Bev were included in the study. All patients were diagnosed with GBM by neuropathologists at our hospital according to the revised 4th edition of the World Health Organization classification scheme [16].

The patient's clinical, operative, and radiological information were reviewed, and the following data were collected for each: clinical history, date of initial operation, postoperative adjuvant therapy regimen, use of corticosteroids at the start of RT, date of tumor recurrence, date of recording a Karnofsky performance status (KPS) score of 50, date of death or last hospital visit, extent of resection, number of TMZ cycles, reason for TMZ discontinuation, and treatment after tumor recurrence. The extent of resection was determined based on the surgeons' operative notes and on postoperative imaging studies, classified as either total if $100 \%$ of the enhanced lesion was resected, subtotal if 95-99\% was resected, partial if $<94 \%$ was resected, or as a biopsy.

\section{Treatment}

After GBM diagnosis, all patients received radiotherapy with concomitant and adjuvant TMZ or TMZ/Bev. Since June 2013, Bev has been approved as a treatment option for GBM, and thereafter upfront TMZ/Bev was administered to patients with KPS scores of 70 or less, as well as patients with KPS scores of 80 who had residual tumors.

The TMZ dose was $75 \mathrm{mg} / \mathrm{m}^{2} /$ day during radiotherapy and $150-200 \mathrm{mg} / \mathrm{m}^{2} /$ day for 5 days every 28 days when administered as adjuvant treatment for a maximum of 24 cycles (until December 2013) or 12 cycles (since January 2014), or until disease progression. The dose of Bev was $10 \mathrm{mg} / \mathrm{kg}$ every 2 weeks or $15 \mathrm{mg} / \mathrm{kg}$ every 3-4 
weeks. Blood testing was performed every week in the concomitant phase, and every month in the adjuvant phase.

All patients received hypofractionated radiotherapy consisting of $45 \mathrm{~Gy}$ at the isocenter in $3 \mathrm{~Gy}$ daily fractions using a 3-dimensional conformal radiotherapy technique with 4 or more portals, including non-coplanar beams in general. Target volumes were determined based on postoperative magnetic resonance imaging (MRI) fused into the treatment planning computed tomography (CT) images; preoperative MRI was also utilized for correlation analysis. The gross tumor volume (GTV) was defined as the residual tumor on contrast-enhanced T1-weighted images. The clinical target volume 1 (CTV1) was defined as the GTV, the surgical cavity, and hyper-intensity area on T2-weighted images or fluid attenuated inversion recovery (FLAIR) images with a $1.5 \mathrm{~cm}$ margin. The CTV2 was defined as the GTV plus the surgical cavity with a 1.5 $\mathrm{cm}$ margin. The planning target volumes 1 and 2 were defined as the CTV1 and CTV2 plus a $0.5 \mathrm{~cm}$ margin, respectively. PTV1 was irradiated up to $30 \mathrm{~Gy}$ in 10 fractions, and boost irradiations of $15 \mathrm{~Gy}$ to the PTV2 were followed. Irradiation fields were modified, if necessary, through dose-volume histogram evaluation for the organs-at-risk such as the brainstem, optic nerve and chiasm, retina, and lens. The maximum dose to the brainstem, retina, optic nerve and chiasm were restricted to 40 Gy. The lens was shielded by multileaf collimators when possible through the beam's eye view.

\section{Response evaluation}

Patients were evaluated using T2-weighted or FLAIR imaging and contrast-enhanced T1-weighted imaging within 3 days before and 1 day after surgery; follow-up imaging via MRI was performed at the time of the completion of RT, 1 month thereafter, and every 2 months thereafter or according to clinical symptom development. When pseudoprogression was suspected and the lesions were stable, the patients were observed without changing adjuvant chemotherapy; patents with lesions that resolved during follow-up were considered to have pseudoprogression.

Disease progression was evaluated according to the Response Assessment Criteria for High-Grade Gliomas (RANO criteria) [17]. Briefly, complete response (CR) was defined as the complete disappearance of all enhancing measurable and non-measurable lesions for at least 4 weeks. Partial response (PR) was defined as a $\geq 50 \%$ decrease in lesion size compared to baseline, as measured by summing the products of the perpendicular diameters of all measurable lesions that were sustained for at least 4 weeks, with no new lesions or any progression of nonmeasurable disease observed. Progressive disease (PD) was defined as $a \geq 25 \%$ increase in the sum of the products of the perpendicular diameters of the enhancing lesions, compared with the smallest tumor measurement, a significant T2-weighted or FLAIR non-enhancing lesion, the appearance of any new lesions, definite clinical deterioration not attributable to causes other than the tumor, or death. If there was uncertainty regarding whether there was progression, close observation or C11-methionine positron emission tomography (Met-PET) was performed. If follow-up imaging studies showed progressive enlargement, tumor recurrence was diagnosed. If low-uptake on Met-PET was observed, then tumor necrosis was diagnosed [18]. Stable disease (SD) was defined has not meeting any of the criteria for CR, PR, or PD. Patients who underwent total resection and did not have measurable lesions were also classified as having SD [17].

\section{IDH1/2 mutation analysis}

Nucleic acids were extracted from tumors using the DNeasy Blood \& Tissue Kit (Qiagen, Maryland, USA), according to the manufacturer's instructions. The mutation hotspots on codon 132 of IDH1 and codon 172 of IDH2 were assessed through pyrosequencing, as previously described [19, 20]. The pyrosequencing assays were designed to detect all known mutations on these codons $[19,20]$.

\section{Immunohistochemistry}

Immunohistochemistry using a mouse monoclonal antihuman isocitrate dehydrogenase 1 (IDH1) $\mathrm{R} 132 \mathrm{H}$ antibody (Dianova, Hamburg, Germany) (clone: H09, dilution 1:50) was performed using the EnVision FLEX system (Dako Japan Inc., Tokyo, Japan) (polymeric method), an automatic staining machine [21].

\section{MGMT promoter methylation analysis}

The methylation status of the MGMT promoter was analyzed using bisulfite modification of the tumor genomic DNA followed by pyrosequencing, as previously described [20]. Methylation was considered positive when its mean level at the examined $16 \mathrm{CpG}$ sites was greater than $16 \%$ [20].

\section{Statistical analysis}

OS was defined as the interval between the date of surgery and that of death. Progression-free survival (PFS) was defined as the interval between the date of surgery and that of the detection of progression. The time until KPS score decrease to below 60 was defined as the interval between the date of surgery to that of the first recording of a KPS score of 50. The patients' OS, PFS, and time until the KPS score dropped below 60 were calculated using the KaplanMeier method and compared using the log-rank test. Analyses were conducted using JMP 8 (SAS Institute Inc., Cary, NC, USA) and GraphPad Prism ${ }^{\circ}$ version 6.0 
(GraphPad Software, La Jolla, California, USA). In all tests, probability values of $<0.05$ were considered statistically significant.

\section{Results}

\section{Patient characteristics and treatments}

The patient characteristics are summarized in Table 1. The median age was 80 years (range: $75-87$ years), and the median KPS score was 70 . Twelve patients (40.0\%) underwent biopsy, and the reasons to perform biopsy included multifocal tumors in 3 patients, deep seated tumors in 5, and eloquent localization in 4 patients. Twenty patients $(66.7 \%)$ received TMZ and 10 (33.3\%) received TMZ/Bev as upfront chemotherapy. The median number of TMZ cycles was 5 (range: 0-20 cycles). Most patients (21 of the 28 patients [75.0\%]) who experienced tumor recurrence terminated treatment and received supportive care only. Twenty-nine tumors were classified as $I D H 1 / 2$ wildtype by pyrosequencing $(N=$ 27) or immunohistochemistry $(N=2)$. Eleven tumors (36.7\%) were classified as having hypermethylation and $16(53.3 \%)$ as having hypomethylation (Table 1$)$.

\section{Outcomes}

The median OS was 12.9 months and the median PFS was 9.9 months (Fig. 1a and b, respectively). The 1-year OS and PFS rates were 64.7 and $34.7 \%$, respectively. Notably, 5 patients lived for more than 2 years after operation. The median OS in patients with MGMT promoter hypermethylation $(N=11)$ was 17.4 months, whereas the median OS in patients with MGMT promoter hypomethylation $(N=16)$ was 11.8 months; the difference was not statistically significant $(p=0.32)$ (Fig. 1c). The median PFS in patients with MGMT promoter hypermethylation and hypomethylation were 13.1 and 7.3 months, respectively; again, the difference was not statistically significant $(p=0.11)$ (Fig. 1d). The median OS and PFS were not significantly different between TMZ $(N=20)$ and TMZ/Bev $(N=10)$ chemotherapy (median OS for TMZ vs. TMZ/Bev: 12.9 months vs. 14.6 months, respectively; $p=0.93$; median PFS for TMZ vs TMZ/Bev: 8.5 months vs. 10.0 months, respectively; $p=0.64$ ) (Fig. 1e and $\mathrm{f}$, respectively). The median time until KPS score decrease to below 60 was 7.9 months (Fig. 2). The best radiological responses were PR in 11 patients (36.7\%), SD in $12(40 \%)$, and PD in 7 (23.3\%); none achieved CR. Pseudoprogression was observed in 1 patient. There was one case, which showed slowly progressive paraventricular disease with lowuptake on Met-PET. We considered this to be a case of radiation necrosis. Three representative patients with PR are described in Fig. 3.

All patients discontinued TMZ or TMZ/Bev maintenance therapy because of tumor progression $(N=10$;
Table 1 Patient characteristics

\begin{tabular}{|c|c|}
\hline & No. (\%) of Patients \\
\hline \multicolumn{2}{|l|}{ Age (yr) } \\
\hline Median & 80 \\
\hline Range & $75-87$ \\
\hline \multicolumn{2}{|l|}{ Sex } \\
\hline Male & $19(63.3)$ \\
\hline Female & $11(36.7)$ \\
\hline \multicolumn{2}{|l|}{ Karnofsky performance status } \\
\hline 100,90 & $2(6.7)$ \\
\hline 80,70 & $17(56.7)$ \\
\hline 60,50 & $11(36.7)$ \\
\hline \multicolumn{2}{|l|}{ Extent of resection } \\
\hline Total resection & $8(26.7)$ \\
\hline Subtotal resection & $3(10)$ \\
\hline Partial resection & $7(23.3)$ \\
\hline Biopsy & $12(40)$ \\
\hline \multicolumn{2}{|l|}{ Chemotherapy } \\
\hline Temozolomide & $20(66.7)$ \\
\hline Temozolomide/Bevacizumab & $10(33.3)$ \\
\hline \multicolumn{2}{|l|}{ Adjuvant Temozolomide cycles (n) } \\
\hline Median & 5 \\
\hline Range & $0-20$ \\
\hline \multicolumn{2}{|l|}{ Use of corticosteroid } \\
\hline Yes & $6(20)$ \\
\hline No & $24(80)$ \\
\hline \multicolumn{2}{|l|}{ Treatment after tumor recurrence } \\
\hline Best supportive care & $21(70)$ \\
\hline Bev & $5(16.7)$ \\
\hline Surgery followed by Temozolomide & $1(3.3)$ \\
\hline Temozolomide & $1(3.3)$ \\
\hline No recurrence & $2(6.7)$ \\
\hline \multicolumn{2}{|l|}{ MGMT promoter status } \\
\hline High & $11(36.7)$ \\
\hline Low & $16(53.3)$ \\
\hline N/A & $3(10)$ \\
\hline \multicolumn{2}{|l|}{ IDH $1 / 2$ status } \\
\hline Wild-type & $29(96.7)$ \\
\hline Mutant & 0 \\
\hline N/A & $1(3.3)$ \\
\hline
\end{tabular}

IDH Isocitrate dehydrogenase, MGMT O6-methylguanine DNA methyltransferase, N/A Not available

33.3\%), clinical deterioration without radiological tumor progression $(N=9,30.0 \%)$, completion of 12 cycles in $(N=3 ; 10.0 \%)$, complications $(N=4 ; 13.3 \%)$, and switching to palliative care at a different hospital $(N=4 ; 13.3 \%)$ (Table 2). 


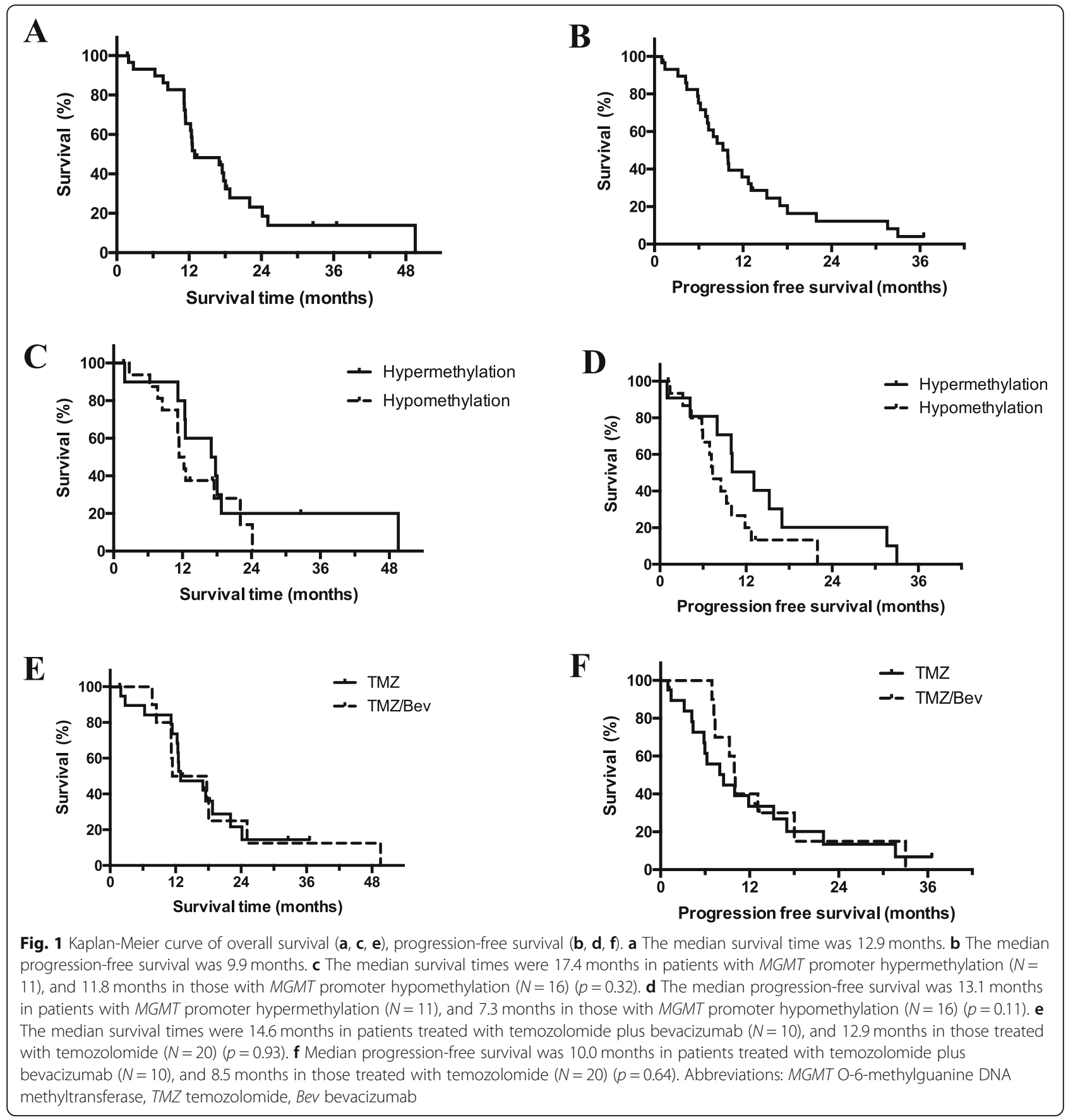

\section{Toxicities}

Toxicities are summarized in Table 3 . In the concomitant phase: 15 patients (50\%) experienced grade 3/4 leukopenia, 4 (13.3\%) had neutropenia, 4 (13.3\%) had anorexia, 3 (10.0\%) had hyponatremia, and 4 (13.3\%) exhibited skin rashes. Among the 24 patients who received adjuvant chemotherapy, 11 (45.8\%) experienced grade 3/ 4 leukopenia, 1 (4.2\%) had neutropenia, 1 (4.2\%) had anemia, and $2(8.3 \%)$ had skin rashes. One patient discontinued TMZ/Bev chemotherapy owing to gastrointestinal hemorrhage. One other patient experienced severe ulceration of the skin of his left leg during TMZ/ Bev chemotherapy and terminated treatment.

\section{Discussion}

The treatment of elderly patients with GBM is challenging. Although TMZ combined with hypofractionated RT of 40 Gy in 15 fractions is established as the standard 


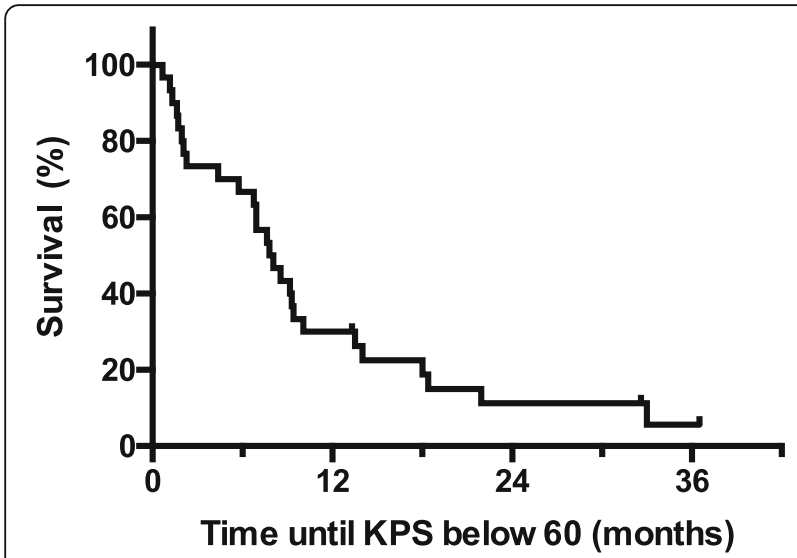

Fig. 2 Kaplan-Meier curve of time until the decrease in Karnofsky performance status score (KPS) to below 60. The median time until KPS score decreased to below 60 was 7.9 months. Abbreviations: KPS Karnofsky performance status

treatment in elderly patients aged $\geq 65$ years, optimal treatment remains unclear, especially in patients aged $\geq 75$ years. The European guidelines state that patients with GBM with $M G M T$-methylation aged $\geq 75$ years may receive hypofractionated RT/TMZ or TMZ alone, and those with GBM without MGMT-methylation may receive hypofractionated $\mathrm{RT}$ alone, indicating that either RT or TMZ alone might be a treatment option [22]. As the elderly population expands, the incidence of GBM in these elderly patients will increase, and the development of effective and safe treatment to improve outcomes in this patient population becomes increasingly important.

A few studies investigated the treatment outcomes in older patients (aged $\geq 75$ years) with GBM. In a phase III trial, Perry et al. showed the survival benefit of adding TMZ to short-course radiotherapy in patients with GBM (aged $\geq 65$ years) and demonstrated the survival benefit of TMZ among patients aged $\geq 76$ years (median OS 10.0 months [RT/TMZ] vs. 7.6 months [RT alone]) [8]. Uzuka et al. analyzed 79 patients with GBM (aged $\geq 76$ years) and reported that the median OS and PFS were 9.8 months and 6.8 months, respectively. Multivariate analysis demonstrated post-operative KPS and TMZ therapy to be significant predictors of survival [23]. Harris et al. retrospectively examined the outcome of 108 patients with GBM (aged $\geq 75$ years) and reported a median OS of 13.3 months, 6.3 months, and 1.9 months in patients who underwent RT/TMZ, RT alone, and best supportive care, respectively. They demonstrated that chemotherapy was the only positive prognostic factor on multivariate analysis and concluded that all patients should be considered for TMZ [24]. Our results that the median OS and PFS were 12.9 and 9.9 months, respectively, and that the 1-year OS and PFS rates were 64.7 and $34.7 \%$, respectively, are comparable with these studies and support the benefit of chemoradiation therapy in these elderly patients.

Various regimens using hypofractionated radiotherapy combined with TMZ for the treatment of elderly patients with GBM have been investigated. Such regimens include 30 Gy in 6 fractions, 40 Gy in 15 fractions, 45 Gy in 15 fractions, and $35 \mathrm{~Gy}$ in 10 fractions. The resulting OS and PFS range intervals were 5.4-20 months, and $3.5-9.6$ months, respectively (Table 4) $[8,13,15,24,25$, $27-29,31,33]$. The biologic radiation response is predicted by using the linear-quadratic model (LQ model); generally, the tumor is considered an acutely responding tissue with high $\alpha / \beta$ ratios, whereas normal tissue (including normal brain) is late-responding with low $\alpha / \beta$ ratios [34]. According to the LQ model, our regimen (45 Gy in 15 fractions) is characterized as having a greater dose-intensity than the regimen of $40 \mathrm{~Gy}$ in 15 fractions, with less impact than the regimen of $60 \mathrm{~Gy}$ in 30 fractions. Terasaki et al. treated 26 patients with TMZ combined with hypofractionated radiotherapy of 45 Gy in 15 fractions and reported median OS and PFS of 15.6 and 9.6 months, respectively, with a response rate of $34.6 \%$. In considering the above together with our result, the regimen of $45 \mathrm{~Gy}$ in 15 fractions seems to be favorable, although it would not be superior to the standard regimen of $60 \mathrm{~Gy}$ in 30 fractions [28]. Roa et al. reported no significant survival differences between 40 Gy in 15 fractions and 25 Gy in 5 fractions in the elderly or frail patients with GBM, suggesting that the $\alpha / \beta$ ratio of GBM could be lower than 2-3 Gy [30]. The low $\alpha / \beta$ ratio of GBM supports the advantage of the hypofractionated approach and further studies are needed to develop the optimal dose-fractionation schedule, which meets efficacy and safety.

Bevacizumab, an antiangiogenic monoclonal antibody, which binds to vascular endothelial growth factor (VEGF), received approval in 2013 as a treatment for both primary and recurrent GBM in Japan. The potential benefits of Bev in elderly patients with GBM have been suggested [11-13]. Babu et al. demonstrated that elderly patients with GBM experienced significantly longer survival after treatment with Bev than those without Bev (20.1 months vs. 7.9 months: $p<0.0001$ ). They also suggested that $\mathrm{Bev}$ could be an independent favorable prognostic factor after adjusting for age, KPS, and extent of resection [11]. Matsuda et al. showed that Bev prolonged overall survival time after tumor recurrence and suggested the usefulness of Bev in a salvage setting in elderly patients with GBM [13]. Although Bev was selectively used in our study for patients with lower performance status or residual tumors, their OS and PFS were comparable to those of patients with higher performance status or extensive tumor removal. This observation suggests the potential benefit of upfront Bev in the elderly patients. 

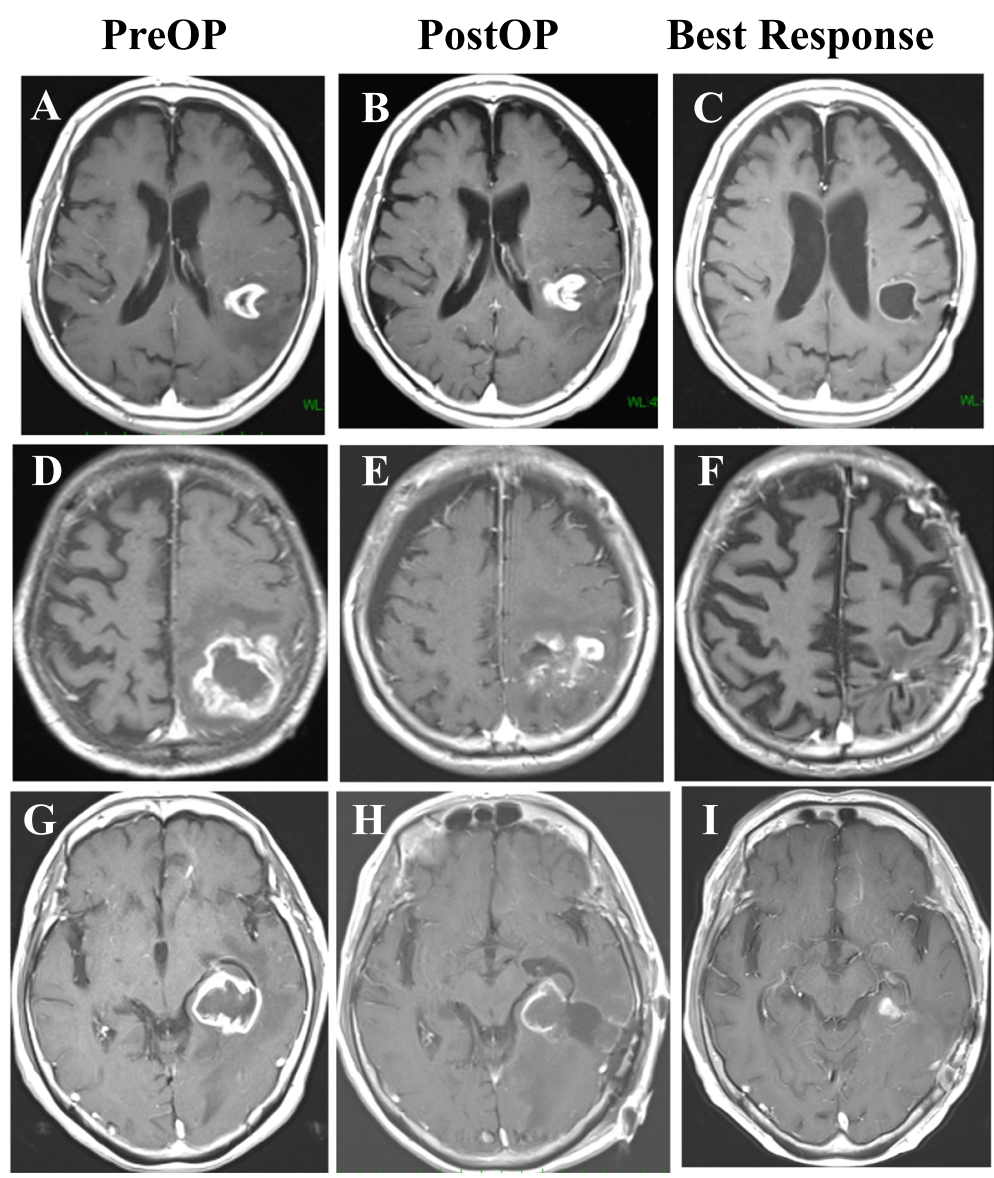

Fig. 3 Three representative patients whose T1-weighted magnetic resonance images with gadolinium enhancement showed a partial response. a An 80-year-old man presented with a left temporo-parietal enhanced tumor and underwent biopsy. $\mathbf{b}$ Afterwards, he received temozolomide combined with radiotherapy consisting of $45 \mathrm{~Gy}$ in 15 fractions and showed a remarkable response with cystic degeneration. c The tumor was MGMT promoter-hypomethylated. d A 78-year-old man presented with a left parietal enhanced tumor and underwent partial resection. e Afterwards, he received temozolomide combined with radiotherapy consisting of $45 \mathrm{~Gy}$ in 15 fractions and nearly achieved a complete response. f The tumor was MGMT promoter-hypermethylated. g A 77-year-old woman presented with a left temporal enhanced tumor and underwent partial resection. $\mathbf{h}$ Afterwards, she received temozolomide combined with radiotherapy consisting of 45 Gy in 15 fractions and achieved tumor size reduction. i The tumor was MGMT promoter-hypomethylated. Abbreviations: MGMT O-6-methylguanine DNA methyltransferase

MGMT promoter methylation status has been reported to be a prognostic factor and a predictor of TMZ treatment efficacy in elderly patients [7, 32]. However, in our study, we found no statistically significant differences in either OS or PFS according to MGMT promoter methylation status, although PFS tended to be longer in patients with MGMT-methylation than in

Table 2 Reasons of temozolomide or temozolomide plus bevacizumab maintenance therapy discontinuation

\begin{tabular}{ll}
\hline & $N=30$ \\
\hline Tumor progression & $10(33.3 \%)$ \\
Clinical deterioration without radiological tumor progression & $9(30.0 \%)$ \\
Completeion of 12 cycles & $3(10.0 \%)$ \\
Complication & $4(13.3 \%)$ \\
Changing hospital for palliative care & $4(13.3 \%)$ \\
\hline
\end{tabular}

Table 3 Summary of Grade 3 or 4 toxicities

\begin{tabular}{lll}
\hline Category & Concomitant $(N=30)$ & Adjuvant $(N=24)$ \\
\hline Hematologic & & \\
Neutropenia & $4(13.3 \%)$ & $1(4.2 \%)$ \\
Lymphocytopenia & $15(50 \%)$ & $11(45.8 \%)$ \\
Thrombocytopenia & $1(3.3 \%)$ & 0 \\
Anemia & $1(3.3 \%)$ & $1(4.2 \%)$ \\
Hepatic & & \\
Aspartate transaminase & $2(6.7 \%)$ & 0 \\
Alanine transaminase & $2(6.7 \%)$ & $1(4.2 \%)$ \\
Pulmonary (pneumonitis) & $2(6.7 \%)$ & $1(4.2 \%)$ \\
Anorexia & $4(13.3 \%)$ & $1(4.2 \%)$ \\
Hyponatremia & $3(10.0 \%)$ & 0 \\
Hypokalemia & $1(3.3 \%)$ & 0 \\
Skin rash & $4(13.3 \%)$ & $1(4.2 \%)$ \\
\hline
\end{tabular}


Table 4 Summary of currently published studies using hypofractionated radiotherapy and temozolomide for elderly patients with glioblastomas

\begin{tabular}{|c|c|c|c|c|c|}
\hline Author & Treatment & $\mathrm{n}$ & Patients & OS (mo) & PFS (mo) \\
\hline Minniti et al. 2009 [33] & RT 30Gy/6fr + TMZ & 43 & Age $\geq 70$ and $K P S \geq 60$ & 9.3 & 6.3 \\
\hline Uto et al. 2015 [31] & RT 35Gy/10fr + TMZ & 11 & Age $\geq 70$ & 13.2 & 7 \\
\hline Minniti et al. 2012 [29] & RT40Gy/15fr + TMZ & 71 & Age $\geq 70$ and $K P S \geq 60$ & 12.4 & 6 \\
\hline \multirow[t]{2}{*}{ Perry et al. 2017 [8] } & RT40Gy/15fr & 281 & Age $\geq 65$ and PS 0-2 & 7.6 & 3.9 \\
\hline & RT40Gy/15fr + TMz & 281 & & 9.3 & 5.3 \\
\hline \multirow[t]{2}{*}{ Lombardi et al. 2015 [28] } & RT40Gy/15fr + TMZ & 71 & Age $\geq 65$ and PS 0-2 & 13.8 & N/A \\
\hline & RT60Gy/30fr + TMz & 166 & & 19.4 & \\
\hline \multirow[t]{2}{*}{ Chang-Halpenny et al. 2015 [25] } & RT 35Gy/10fr + TMZ & 29 & Age $\geq 65$ & 5.4 & N/A \\
\hline & RT60Gy/30fr + TMz & 100 & & 13 & \\
\hline Terasaki et al. 2011 [15] & RT45Gy/15fr + TMZ & 26 & median 61(39-79) & 15.6 & 9.6 \\
\hline Lim et al. 2015 [27] & RT45Gy/15fr + TMZ & 33 & $\begin{array}{l}\text { Age } \geq 70 \text { Age }<70 \text { and } P S \geq 3 \text { or } \\
\text { biopsy or rapid growth }\end{array}$ & 10.6 & 7.5 \\
\hline \multirow[t]{3}{*}{ Harris et al. 2017 [24] } & Best supportive care & 31 & Age $\geq 75$ & 1.9 & N/A \\
\hline & RT alone* & 38 & & 6.2 & N/A \\
\hline & $R T^{*}+\mathrm{TMZ}$ & 33 & & 13.2 & N/A \\
\hline Matsuda et al. 2018 [13] & RT45Gy/15fr + TMZ, Bev after recurrence & 18 & Age $\geq 75$ & 20 & 2.5 \\
\hline Present study & RT45Gy/15fr + TMZ or TMZ/Bev & 30 & Age $\geq 75$ & 12.9 & 9.9 \\
\hline
\end{tabular}

RT Radiation therapy, TMZ Temozolomide, Bev Bevacizumab, PFS Progression free survival, OS Overall survival, KPS Karnofsky performance status, PS Performance status, N/A not available

* Radiation therapy was either hypofractionation (40Gy), or longer-course (60Gy)

those without. Similarly, Biau et al. reported that $M G M T$ promoter methylation was neither a prognostic nor a predictive factor in a real-life, unselected elderly patient population [35]. In our study, the small number of patients and intratumoral heterogeneity [36] may explain the lack of statistical significance. Our results suggest the potential benefit of using TMZ, irrespective of $M G M T$ promoter methylation status.

In our cohort, we had 5 patients who lived for more than 2 years. The possible reasons of these favorable outcomes might be multifactorial, including total removal, high performance status, upfront Bev, and MGMT hyper methylation. Our cases suggest that there might be some patients who have great benefit from the aggressive treatment in spite of their advanced age.

The quality of life or in-house independence is an important treatment goal for elderly patients with GBM. Chinot et al. reported that the median time of maintaining a KPS score of 70 or higher in adult patients with GBM was 9.0 months in their radiotherapy/TMZ/Bevtreated group and 6.0 months in their radiotherapy/ TMZ-treated group [37]. In comparison, the median time for maintaining a KPS score of 60 or higher in our study was 7.9 months, which seems to be favorable in taking into consideration their elderly patient population. Our results suggest that our treatment approach could enable elderly patients to maintain their activities of daily living for longer periods.
Nine patients terminated TMZ or TMZ/Bev maintenance treatment owing to clinical deterioration without radiological evidence of tumor progression, as did 4 owing to complications. Clinical deterioration and the development of complications are difficult to predict before treatment; moreover, most patients in our study (75\%) terminated treatment after their first tumor recurrence and received only supportive care. These observations exemplify the vulnerability of elderly patients. To minimalize the treatment risk, such vulnerabilities must be investigated, and appropriate selection criteria must be devised to better identify patients with treatmentrelated vulnerabilities.

Treatment-related toxicities are a main concern for elderly patients treated for GBM. We observed grade 3/4 leukopenia in half of our patients; $10-13 \%$ of our patients also experienced anorexia, hyponatremia, and skin rash during TMZ or TMZ/Bev treatment. All these toxicities were manageable, and anorexia was related to the TMZ; hyponatremia and hypokalemia were related to the anorexia. Skin rashes in 4 patients were assumed to be drug-related (i.e due to an anticonvulsant), and one skin rash was caused by Herpes Zoster virus infection. Thrombocytopenia was observed in only one patient, which is lower than that of the other study by Perry, et al. [8]. Two of our patients also experienced severe non-hematological toxicities (gastrointestinal hemorrhage, and skin ulceration, respectively), which we assumed to be 
a consequence of Bev administration. As such, physicians should remain cognizant of the potential risk of these uncommon but severe complications, and thus observe their patients (especially the elderly among them) more carefully.

Our study had certain limitations. First, this work was retrospective, and we did not provide data regarding the quality of life during the course of treatment. Second, our cohort was too small to draw definitive conclusions; however, most of our patients were followed from the time of their surgeries to their terminal stages at our institute, thereby providing robust clinical data.

In conclusion, our hypofractionated radiotherapy and upfront Bev combined with TMZ approach appears to be feasible with favorable outcomes and acceptable toxicities in patients aged $\geq 75$ years. Further studies are needed to develop the optimal dose-fractionation schedule and combination with chemotherapy in the elderly patients with GBM.

\section{Abbreviations}

Bev: Bevacizumab; CR: Complete response; CTV: Clinical target volume; FLAIR: Fluid-attenuated inversion recovery; GBM: Glioblastoma; GTV: Gross tumor volume; IDH: Isocitrate dehydrogenase; KPS: Karnofsky performance status; MGMT: O-6-methylguanine DNA methyltransferase; MMSE: MiniMental State Examination score; MRI: Magnetic resonance imaging; OS: Overall survival; PD: Progressive disease; PFS: Progression-free survival; PR: Partial response; RANO: Response Assessment Criteria for High-Grade Gliomas; SD: Stable disease; TMZ: Temozolomide

\section{Acknowledgements}

Not applicable

\section{Authors' contributions}

$\mathrm{MO}$ and $\mathrm{YN}$ designed the study. MO, YM, MT, HI, and YN contributed to patients treatment and management. YM and $\mathrm{KI}$ identified IDH1/2 mutations and MGMT status. MO and YN interpreted the data and performed the biostatistical analysis. $\mathrm{MO}$, and $\mathrm{HI}$ wrote the manuscript. All the authors reviewed and approved this manuscript.

\section{Funding}

None to report.

\section{Availability of data and materials}

The datasets used and/or analyzed during the current study are available from the corresponding author on reasonable request.

\section{Ethics approval and consent to participate}

All procedures performed in this study were in accordance with the ethical standards of the institutional and the 1964 Helsinki declaration and its later amendments. This study was approved by the internal review board of the National Cancer Center (2004-066 or 2007-086). Written informed consent was obtained from all individual participants.

\section{Consent for publication}

Not applicable.

\section{Competing interests}

All the authors have nothing to disclose except Dr. Ichimura and Dr. Narita. Dr. Ichimura reports grants from Chugai Pharmaceutical Co., Ltd., Eisai Co. Ltd., and Daiichi Sankyo Co.Ltd., outside the submitted work. Dr. Narita reports grants from Japan Agency for Medical Research and Development, Chugai Pharmaceutical co., MSD, Eisai, Toshiba, SBI pharma, Glaxo, Abbive, Ono, Stella-pharma, Ohtuka, Meiji-seika, and Daiichi-Sankyo, outside the submitted work.

\section{Author details}

Department of Neurosurgery and Neuro-Oncology, National Cancer Center Hospital, 5-1-1, Tsukiji, Chuo-ku, Tokyo 104-0045, Japan. ²Department of Radiation Oncology, National Cancer Center Hospital, 5-1-1, Tsukiji, Chuo-ku, Tokyo 104-0045, Japan. ${ }^{3}$ Division of Brain Tumor Translational Research, National Cancer Center Research Institute, 5-1-1, Tsukiji, Chuo-ku, Tokyo 104-0045, Japan.

Received: 6 June 2019 Accepted: 2 October 2019

Published online: 12 November 2019

\section{References}

1. Committee_of_Brain_Tumor_Registry_of_Japan. Report of Brain Tumor Registry of Japan (2005-2008) 14th Edition. Neurol Med Chir (Tokyo). 2017; 57:9-102.

2. Laperriere N, Weller M, Stupp R, Perry JR, Brandes AA, Wick W, et al. Optimal management of elderly patients with glioblastoma. Cancer Treat Rev. 2013; 39:350-7.

3. Paszat L, Laperriere N, Groome P, Schulze K, Mackillop W, Holowaty E. A population-based study of glioblastoma multiforme. Int J Radiat Oncol Biol Phys. 2001;51:100-7

4. Ostrom QT, Gittleman H, Liao P, Vecchione-Koval T, Wolinsky Y, Kruchko C, et al. CBTRUS statistical report: primary brain and other central nervous system tumors diagnosed in the United States in 2010-2014. NeuroOncology. 2017;19:v1-v88.

5. Hingorani M, Colley WP, Dixit S, Beavis AM. Hypofractionated radiotherapy for glioblastoma: strategy for poor-risk patients or hope for the future? $\mathrm{Br}$ J Radiol. 2012;85:e770-81.

6. Roa W, Brasher PM, Bauman G, Anthes M, Bruera E, Chan A, et al. Abbreviated course of radiation therapy in older patients with glioblastoma multiforme: a prospective randomized clinical trial. J Clin Oncol. 2004;22:1583-8.

7. Malmstrom A, Gronberg BH, Marosi C, Stupp R, Frappaz D, Schultz H, et al. Temozolomide versus standard 6-week radiotherapy versus hypofractionated radiotherapy in patients older than 60 years with glioblastoma: the Nordic randomised, phase 3 trial. Lancet Oncol. 2012;13:916-26.

8. Perry JR, Laperriere N, O'Callaghan CJ, Brandes AA, Menten J, Phillips C, et al. Short-course radiation plus Temozolomide in elderly patients with Glioblastoma. N Engl J Med. 2017:376:1027-37.

9. Minniti G, Lombardi G, Paolini S. Glioblastoma in Elderly Patients: Current Management and Future Perspectives. Cancers (Basel). 2019;11(3):336. https://doi.org/10.3390/cancers11030336

10. Sulman EP, Ismaila N, Armstrong TS, Tsien C, Batchelor T, Cloughesy T, et al. Radiation therapy for Glioblastoma: American Society of Clinical Oncology clinical practice guideline endorsement of the American Society for Radiation Oncology guideline. J Clin Oncol. 2017;35:361-9.

11. Babu R, Komisarow JM, Agarwal VJ, Rahimpour S, lyer A, Britt D, et al. Glioblastoma in the elderly: the effect of aggressive and modern therapies on survival. J Neurosurg. 2016;124:998-1007.

12. Davies J, Reyes-Rivera I, Pattipaka T, Skirboll S, Ugiliweneza B, Woo S, et al. Survival in elderly glioblastoma patients treated with bevacizumab-based regimens in the United States. Neurooncol Pract. 2018;5:251-61.

13. Matsuda Kl, Sakurada K, Nemoto K, Kayama T, Sonoda Y. Treatment outcomes of hypofractionated radiotherapy combined with temozolomide followed by bevacizumab salvage therapy in glioblastoma patients aged > 75 years. Int J Clin Oncol. 2018;23:820-5.

14. Nghiemphu PL, Liu W, Lee Y, Than T, Graham C, Lai A, et al. Bevacizumab and chemotherapy for recurrent glioblastoma: a single-institution experience. Neurology. 2009;72:1217-22.

15. Terasaki M, Eto T, Nakashima S, Okada Y, Ogo E, Sugita Y, et al. A pilot study of hypofractionated radiation therapy with temozolomide for adults with glioblastoma multiforme. J Neuro-Oncol. 2011;102:247-53.

16. Louis DN, Ohgaki H, Wiestler OD, WK C. WHO classification of tumours of the central nervous system. Revise 4th edition. Lyon: IARC Press; 2016

17. Wen PY, Macdonald DR, Reardon DA, Cloughesy TF, Sorensen AG, Galanis E, et al. Updated response assessment criteria for high-grade gliomas: response assessment in neuro-oncology working group. J Clin Oncol. 2010; 28:1963-72.

18. luchi T, Hatano K, Kodama T, Sakaida T, Yokoi S, Kawasaki K, et al. Phase 2 trial of hypofractionated high-dose intensity modulated radiation therapy 
with concurrent and adjuvant temozolomide for newly diagnosed glioblastoma. Int J Radiat Oncol Biol Phys. 2014;88:793-800.

19. Saito K, Mukasa A, Narita Y, Tabei Y, Shinoura N, Shibui S, et al. Toxicity and outcome of radiotherapy with concomitant and adjuvant temozolomide in elderly patients with glioblastoma: a retrospective study. Neurol Med Chir (Tokyo). 2014;54:272-s.

20. Arita H, Narita Y, Fukushima S, Tateishi K, Matsushita Y, Yoshida A, et al. Upregulating mutations in the TERT promoter commonly occur in adult malignant gliomas and are strongly associated with total 1p19q loss. Acta Neuropathol. 2013;126:267-76.

21. Arita H, Narita Y, Matsushita Y, Fukushima S, Yoshida A, Takami H, et al. Development of a robust and sensitive pyrosequencing assay for the detection of IDH1/2 mutations in gliomas. Brain Tumor Pathol. 2015;32(1): 22-30.

22. Weller M, Le Rhun E, Preusser M, Tonn JC, Roth P. How we treat glioblastoma. ESMO Open. 2019;4:e000520.

23. Uzuka T, Asano K, Sasajima T, Sakurada K, Kumabe T, Beppu T, et al. Treatment outcomes in glioblastoma patients aged 76 years or older: a multicenter retrospective cohort study. J Neuro-Oncol. 2014;116:299-306

24. Harris G, Jayamanne D, Wheeler H, Gzell C, Kastelan M, Schembri G, et al. Survival outcomes of elderly patients with Glioblastoma Multiforme in their 75th year or older treated with adjuvant therapy. Int J Radiat Oncol Biol Phys. 2017;98:802-10.

25. Chang-Halpenny CN, Yeh J, Lien WW. Elderly patients with glioblastoma multiforme treated with concurrent temozolomide and standard- versus abbreviated-course radiotherapy. Perm J. 2015;19:15-20.

26. Keime-Guibert F, Chinot O, Taillandier L, Cartalat-Carel S, Frenay M, Kantor G, et al. Radiotherapy for glioblastoma in the elderly. N Engl J Med. 2007:356:1527-35.

27. Lim YJ, Kim IH, Han TJ, Choi SH, Park SH, Park CK, et al. Hypofractionated chemoradiotherapy with temozolomide as a treatment option for glioblastoma patients with poor prognostic features. Int J Clin Oncol. 2015;20:21-8.

28. Lombardi G, Pace A, Pasqualetti F, Rizzato S, Faedi M, Anghileri E, et al. Predictors of survival and effect of short (40 Gy) or standard-course (60 Gy) irradiation plus concomitant temozolomide in elderly patients with glioblastoma: a multicenter retrospective study of AINO (Italian Association of Neuro-Oncology). J Neuro-Oncol. 2015;125:359-67.

29. Minniti G, Lanzetta G, Scaringi C, Caporello P, Salvati M, Arcella A, et al. Phase II study of short-course radiotherapy plus concomitant and adjuvant temozolomide in elderly patients with glioblastoma. Int J Radiat Oncol Biol Phys. 2012;83:93-9.

30. Roa W, Kepka L, Kumar N, Sinaika V, Matiello J, Lomidze D, et al: International Atomic Energy Agency randomized phase III study of radiation therapy in elderly and/or frail patients with newly diagnosed Glioblastoma Multiforme. J Clin Oncol. 2015:33:4145-50

31. Uto M, Mizowaki T, Ogura K, Arakawa Y, Mineharu Y, Miyamoto S, et al. Feasibility evaluation of hypofractionated radiotherapy with concurrent temozolomide in elderly patients with glioblastoma. Int J Clin Oncol. 2016;21:1023-9.

32. Wick W, Platten M, Meisner C, Felsberg J, Tabatabai G, Simon M, et al. Temozolomide chemotherapy alone versus radiotherapy alone for malignant astrocytoma in the elderly: the NOA-08 randomised, phase 3 trial. Lancet Oncol. 2012;13:707-15.

33. Minniti G, De Sanctis V, Muni R, Rasio D, Lanzetta G, Bozzao A, et al. Hypofractionated radiotherapy followed by adjuvant chemotherapy with temozolomide in elderly patients with glioblastoma. J Neuro-Oncol. 2009:91:95-100

34. Amelio D, Lorentini S, Schwarz M, Amichetti M. Intensity-modulated radiation therapy in newly diagnosed glioblastoma: a systematic review on clinical and technical issues. Radiother Oncol. 2010;97:361-9.

35. Biau J, Chautard E, De Schlichting E, Dupic G, Pereira B, Fogli A, et al. Radiotherapy plus temozolomide in elderly patients with glioblastoma: a "real-life" report. Radiat Oncol. 2017:12:197.

36. Parker NR, Hudson AL, Khong P, Parkinson JF, Dwight T, Ikin RJ, et al. Intratumoral heterogeneity identified at the epigenetic, genetic and transcriptional level in glioblastoma. Sci Rep. 2016;6:22477.

37. Chinot OL, Wick W, Mason W, Henriksson R, Saran F, Nishikawa R, et al. Bevacizumab plus radiotherapy-temozolomide for newly diagnosed glioblastoma. N Engl J Med. 2014;370:709-22.

\section{Publisher's Note}

Springer Nature remains neutral with regard to jurisdictional claims in published maps and institutional affiliations.

Ready to submit your research? Choose BMC and benefit from:

- fast, convenient online submission

- thorough peer review by experienced researchers in your field

- rapid publication on acceptance

- support for research data, including large and complex data types

- gold Open Access which fosters wider collaboration and increased citations

- maximum visibility for your research: over $100 \mathrm{M}$ website views per year

At BMC, research is always in progress.

Learn more biomedcentral.com/submissions 